\title{
The Mapping of the Measured Cardiac Electric Potential and Magnetic-Field Distribution (").
}

\author{
M. J. Peters, Z. Dunajski, A. Heringa and R. Th. van Dam \\ Twente University of Technology, Department of Technical Physics \\ P.O.B. 217, 7500 AE Enschede, The Netherlands
}

(ricevuto il 12 Novembre 1982)

Summary. - The distribution of both the cardiac electric potential on the body surface and the cardiac magnetic field near the chest and back is recorded at fixed time instants of the heart cycle. The general features of both types of maps are simple and reproducible, moreover they both reveal different information.

PACS. 87.40. - Biomagnetism (including magnetocardiography).

PACS. 85.25. - Superconducting devices; superconducting magnets.

\section{1. - Introduction.}

The electric generators in the human heart give rise to electrocardiograms and magnetocardiograms. The information contained in ECGs and MCGs is interdependent, although the possibility that a measurable magnetic field is generated by sources giving no electric field (vortex sources) cannot be excluded. However, the two measuring methods emphasize different aspects of the underlying electric activity. Therefore, it is worthwhile studying the registration of both types of cardiograms.

There are several ways to present measurements of the electric potential and the magnetic field. The potential distribution over the thoracic wall at fixed time instants can be displayed by plotting the isopotential lines on a map of the body surface. By generating these maps for successive time instants,

(*) Paper presented at the "IV International Workshop on Biomagnetism ", held in Rome, September 14-16, 1982. 
e.g. $4 \mathrm{~ms}$ apart, a complete account of the potentials on the body throughout the cardiac cycle can be given. This body surface mapping has proved to be a valuable tool for the recognition of disturbances in the electrical activity of the heart $(1,2)$.

The distribution of a component of the magnetic field can be given in an analogous way by plotting the isomagnetic field component lines on a chosen surface outside the body. Conventional recorded MCGs (i.e. the registration of a component of the magnetic field in a certain point as a function of time) turn out to be poorly reproducible. The amplitude of the $Q R S$ wave changes significantly when the subject of the experiment changes his posture, keeping the position of the cryostat relative to the chest wall constant. The general features of isomagnetic field component maps are not very dependent on the posture of the subject. Maps are suitable for comparison of both measured and simulated ECG and MCG data. The presence of maxima and minima in both maps forms a visual aid which may help theoretical evaluation. Maps of both the electric potential and the magnetic field give the most complete information on the electrical activity of the heart that can be obtained noninvasively.

A considerable amount of previous work on electrocardiography is related to the question of which and how many body surface locations should be measured $\left({ }^{3}\right)$. At the University of Nijmegen 64 leads are chosen and the ECGs are measured simultaneously. At the Twente University of Technology the vertical component of the magnetic field is measured consecutively at 30 points on each of the two parallel frontal planes, one plane at $2 \mathrm{~cm}$ from the sternum and the other at $2 \mathrm{~cm}$ from the spine.

\section{2. - The distribution of the electric potential and the magnetic field as a result of current dipoles.}

Bioelectric sources generate an electric field which can be described as resulting from electric-current dipoles situated in a conductive body. The magnetic field is produced by the same sources plus vortex sources, which may be measurable but which are not taken into account. In this section we discuss the pattern of magnetic-field and electric-potential maps due to various dipole arrangements in order to become familiar with some major features of such maps.

(1) B. Taccardi, L. De Ambroggi and C. Viganotti: in The Theoretical Basis of Electrocardiology, edited by C. V. Nelson and D. B. Geselowitz (Oxford, 1976), Chapter 19.

(2) G. S. SoHI and N. C. Flowers: Circulation, 60, 1354 (1979).

(a) R. C. Barr, M. S. Spach and G. Scott Herman-Giddens: IEEE Trans. Bio-

Med. Eng., BME-18, 2 (1971). 
A current dipole $\boldsymbol{p}=\left(p_{\boldsymbol{x}}, p_{y}, p_{z}\right)$ placed in an infinite homogeneous conductor with a specific conductivity $\sigma$ gives rise to an electric potential and a magnetic induction with

$$
V=\frac{\boldsymbol{p} \cdot \mathbf{r}}{4 \pi \sigma r^{3}} \text { and } \boldsymbol{B}=\mu_{0} \frac{\boldsymbol{p} \times \mathbf{r}}{4 \pi r^{3}},
$$

where $r$ is the distance from the source to the point of observation. From the first formula it follows that the pattern of isopotential lines is the same as the one resulting for a charge dipole.

We take one component of the magnetic field (the $x$-component). This means that only the contribution of the components of a current dipole in the $(y, z)$-plane is seen because

$$
B_{x}=\mu_{0} \frac{\boldsymbol{p} \times \boldsymbol{r}}{4 \pi r^{3}} \cdot \boldsymbol{e}_{x}=\mu_{0} \frac{p_{y} z-p_{x} y}{4 \pi r^{3}}
$$

The isofunction maps of $B_{x}$ in a $(y, z)$-plane for the three components of a single

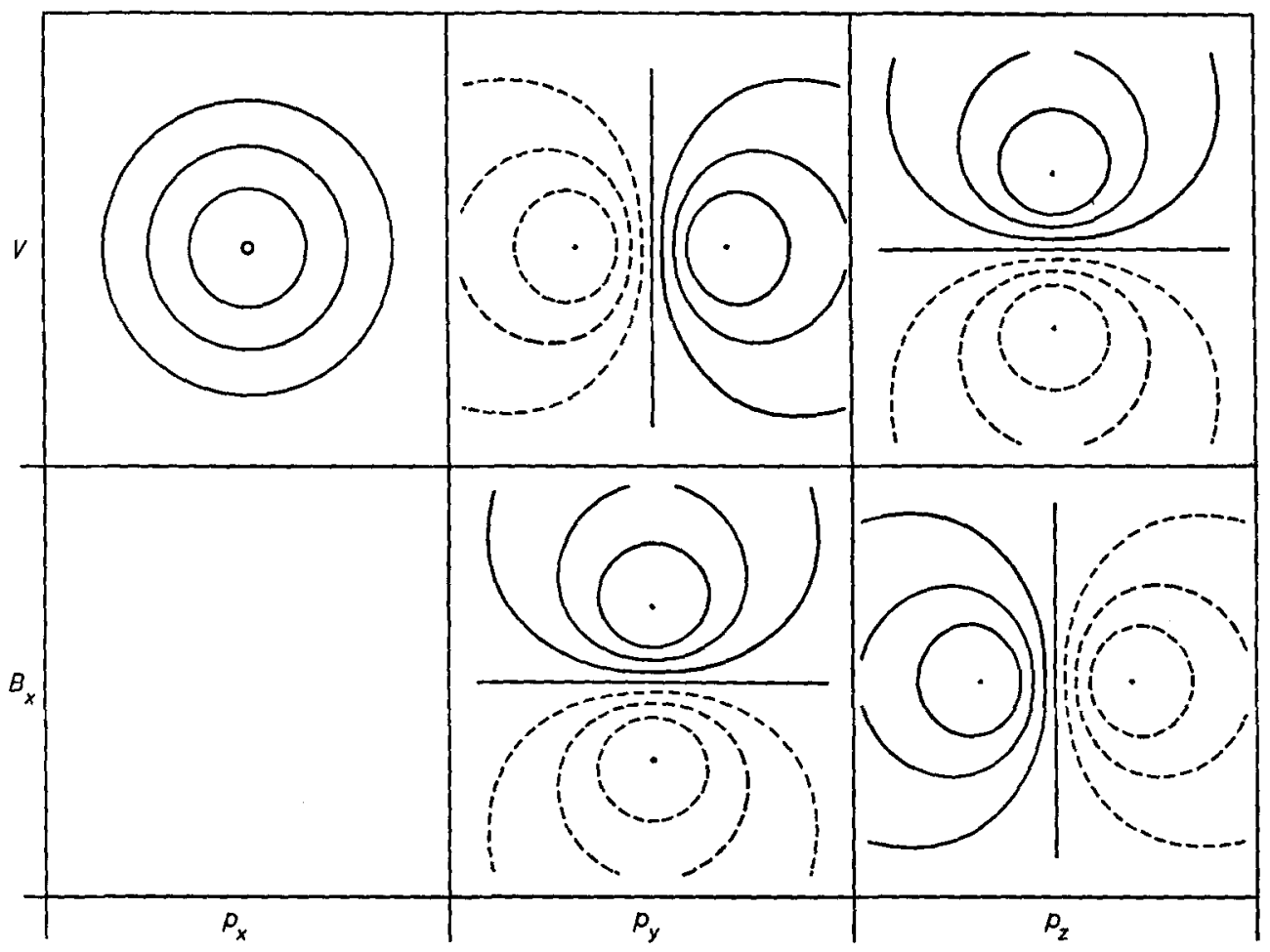

Fig. 1. - Isofunction plots of $B_{x}$ and $\nabla$ in a $(y, z)$-plane for the three components of a single dipole $\boldsymbol{p}=\left(p_{x}, p_{y}, p_{z}\right)$ placed in a homogeneous volume conductor of infinite extent. 
dipole are given in fig. 1. This figure also represents the accessory isopotential maps. It is clear that, when the isopotential map shows circles, the isomagnetic field map for $B_{x}$ is blank. The lines indicating zero value in the isomagnetic field map and isopotential map for a single dipole in a $(y, z)$-plane are perpendicular to each other. The resultant pattern in the isofunction maps produced by a number of current dipoles is a summation of patterns as given in fig. 1 (all patterns may have different scales).

The volume conduction will influence both the magnetic field and the electric potential maps. In fig. 2a) a single dipole $\boldsymbol{p}=p \boldsymbol{e}_{z}$ in the same position as the one taken in fig. 1 is placed in a homogeneous medium bounded by a realistically shaped model of the torso. We look at the magnetic-field component perpendicular to a frontal plane (i.e. a $(y, z)$-plane) near the chest. As is shown, the symmetry in the configuration is lessened, although the original character of the map is preserved. In fig. $2 b$ ) the dipole $\boldsymbol{p}=p \boldsymbol{e}_{z}$ is placed deeper within the torso; in this case the influence of the volume conductor is quite impressive.

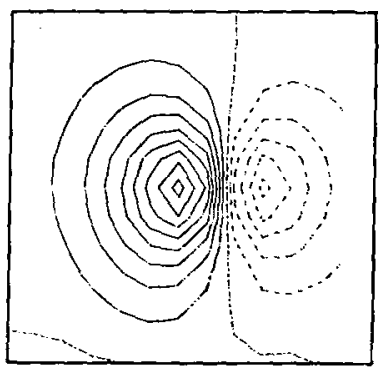

a)

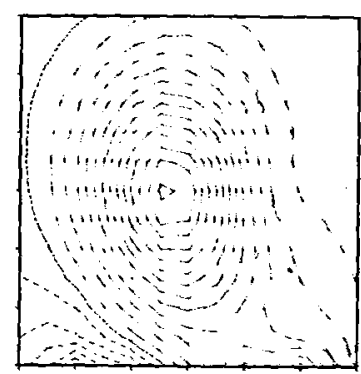

b)

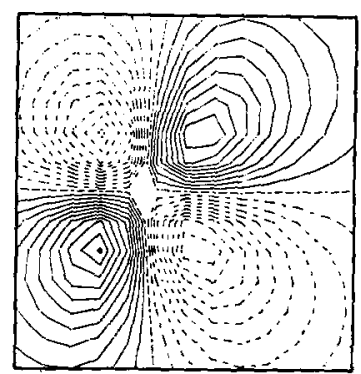

c)

Fig. 2. - Isofunction plot of $B_{x}$ in the $(x=0)$-plane, which is a frontal plane at $2 \mathrm{~cm}$ from the sternum. a) The dipole $\boldsymbol{p}=p \boldsymbol{e}_{z}$ is placed at $x=7 \mathrm{~cm}$ in a homogeneous volume conductor bounded by a realistically shaped model of the torso. b) The dipole $\boldsymbol{p}=p \boldsymbol{e}_{z}$ is placed at $x=15 \mathrm{~cm}$. c) Isofunction plot of $B_{y}$ for the dipole $\boldsymbol{p}=p \boldsymbol{e}_{\boldsymbol{y}}$ taken in the plane $x=7 \mathrm{~cm}$.

The effect of the volume conductor on different components of the magnetic. field was found to be minimal for the normal component on the body surface $\left({ }^{4}\right)$, which is very difficult to measure. Fortunately, the influence on the component of the field perpendicular to a frontal plane, $B_{x}$, has the same order of magnitude $\left({ }^{5}\right)$. Figure $2 c$ ) is an illustration of the influence of the volume conductor on $B_{y}$ for a dipole $\boldsymbol{p}=p \boldsymbol{e}_{y}$ taken in the same position as the one in fig. $2 a$ ). The number of maxima and minima is doubled.

(4) B. M. HoraceK: IEEE Trans. Magn., MAG-9, 3 (1973).

(5) M. J. PETER: The detection of bioelectric sources within the heart using a SQUID sensor. Thesis, Enschede, The Netherlands (1981). 


\section{3. - Measuring methods.}

The electric potential is measured (with Wilson's Central Terminal as a reference) at 64 sites distributed irregularly over the thorax. The signals are amplified by a factor of 2000 by means of isolation amplifiers, low-pass filtered at $250 \mathrm{~Hz}$, by using a 4th-order Bessel filter, fed into 64 sample and hold units and $A D$ converted at the rate of 500 samples/s. The range of the amplifiers is $\pm 5 \mathrm{mV}$ referred to the input. The resolution is $2.5 \mu \mathrm{V}$, which is somewhat less than the noise of the amplifiers ( $3 \mu V$ r.m.s.). The data acquisition is achieved under control of a DEC PDP 11/34. More details can be found in ref. $\left({ }^{6}\right)$.

The isomagnetic maps are constructed from MCGs measured at 30 sites in a frontal plane $2 \mathrm{~cm}$ from the sternum and at 30 sites in a parallel plane $2 \mathrm{~cm}$ from the back bone. The distribution of measuring points should correspond with the density of field lines. This density was unknown, therefore we took a regular grid with points $4 \mathrm{~cm}$ apart. The site of the measuring points is not reflected in isofunction maps. However, in the future we plan to include the grid points as suggested by SAARINEN et al. $\left({ }^{\circ}\right)$, because this will enable us to compare conventional displayed MCGs. The subject was in a horizontal position. The recording was performed in a normal laboratory by using a SQUID magnetometer. The magnetic signal is coupled into the SQUID by means of a symmetric second-order gradiometer with a baseline of $6 \mathrm{~cm}$, the area of the loop was a circle with a diameter of $3 \mathrm{~cm}$. The SQUID output was led via a preamplifier (a SHE $300 \mathrm{RF}$ head) to an electronic processing unit (SHE $330 \mathrm{X}$ control unit). The resulting voltage variations form a measure of the variations in the magnetic flux through all loops of the gradiometer with respect to a constant unknown level.

The total flux divided by the area of the pick-up loop is defined as the effective magnetic induction $B_{\text {eff }}$. This quantity is mapped in the form of isomagnetic field component lines.

Although the SQUID magnetometer used is able to measure visually evoked responses, the cardiac magnetic field is very small in certain positions (especially near the back) at certain time instants of the heart cycle. For some subjects the distance between certain measuring points in a horizontal plane and the body surface is several centimetres. Therefore, we had to diminish the noise, i.e. disturbances from various sources.

${ }^{6}{ }^{6}$ A. Heringa, G. J. H. Uyen and R. Th. VAn Dam: Proceedings of the VIII International Congress on Electrocardiology (Budapest, 1981).

$\left({ }^{3}\right)$ M. Saarinen, P. Sildtanen, P. J. Karp and T. E. Katila: Ann. Clin. Res., 10, 21 (1978). 
4. - Signal processing of the MCGs.

The signal processing with the help of a LSI 11 minicomputer has two main functions: the reduction of noise and the construction of maps.

For the construction of a map we have to know the value of $B_{\text {eff }}$ (with respect to a certain level) at a well-defined time instant. Because all higher-order analogue filters give a frequency-dependent time delay, we used digital filters

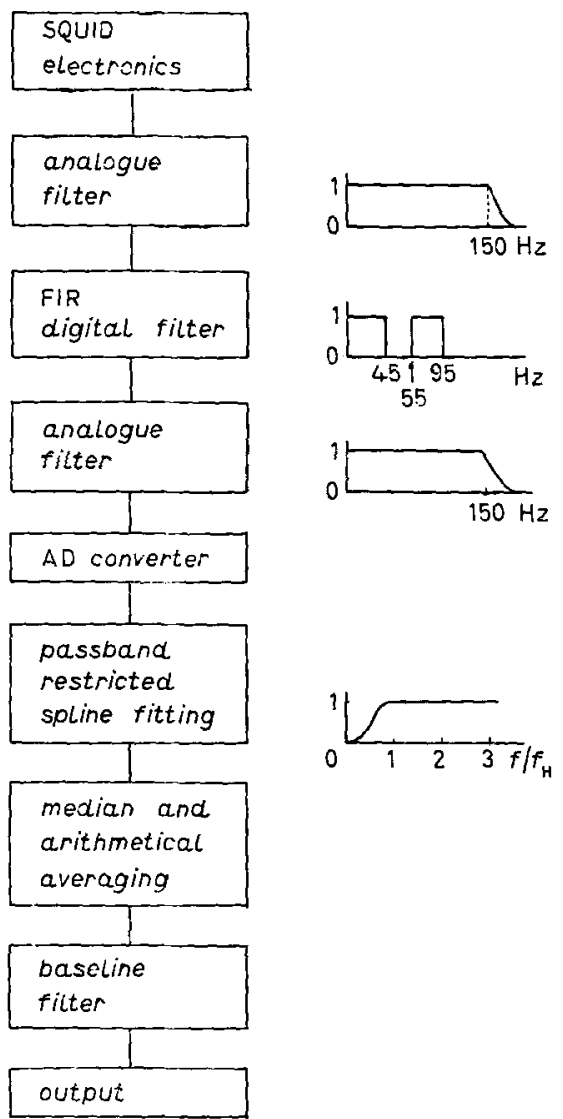

Fig. 3. - Flow chart of the processing system used.

which give a known frequency-independent time delay. The flow chart of the filter system used is given in fig. 3. This system is based on the following arguments:

1) A power spectrum analysis of the MCG emphasized a high concentration of power at low frequencies. 
2) Experiments with different pass bands around $50 \mathrm{~Hz}$ showed that the removal of the frequencies between 46 and $54 \mathrm{~Hz}$ did not noticeably change the shape of the wares in the MCG.

3) We assume that for healthy subjects there is no information contained in the MCGs below the heart beat frequency $(\approx 1 \mathrm{~Hz})$.

The advantage of filtering the frequencies below $1 \mathrm{~Hz}$ is obvious, we want to avoid baseline fluctuations, for instance provoked by the breathing of the subject of the experiment.

The FIR filter (finite-impulse response filter) is a specially designed digital filter with a Kaiser window and a sampling frequency of $500 \mathrm{~Hz}$. It is used as band filter for the removal of the frequencies around $50 \mathrm{~Hz}$ and a slow-pass filter at $95 \mathrm{~Hz}$. The attenuation at $50 \mathrm{~Hz}$ is $60 \mathrm{~dB}$. This filter is preceded by a $48 \mathrm{~dB} /$ octave low-pass Butterworth filter with roll-off at $150 \mathrm{~Hz}$, in order to avoid reconstruction errors. The high-pass filtering is carried out in the LSI 11 minicomputer by means of a so-called pass band restricted spline-fitting algorithm. This gave an attenuation of $3 \mathrm{~dB}$ for the heart beat frequency and more than $60 \mathrm{~dB}$ for $0 \mathrm{~Hz}$. The sampling rate for the $\mathrm{AD}$ conversion was $250 \mathrm{~Hz}$ and we used a $24 \mathrm{~dB} /$ octave low-pass Butterworth filter with roll-off at $150 \mathrm{~Hz}$ as presampling filter.

For each lead we had an acquisition of 16 beats. All the MCGs are stored in a computer memory. To synchronize the signal-averaging process and to provide a common time reference for MCGs recorded at different locations, a trigger pulse was derived from a simultaneously recorded ECG (lead II). The $R$-top from this lead is taken as the time reference and the single ECGs are shifted in time for maximal correlation between $Q R S$ complexes.

The values of the 16 stored MCG signals are sized at 4 ms intervals and the 3 highest and the 3 lowest values at each time instant of the heart cycle are removed (so-called median averaging). The remaining 10 signals are arithmetically averaged, which means that they are superimposed and then divided by 10. Calculations show that the signal processing technique mentioned in this section does not distort the MCGs more than $1 \%$. The signal-to-noise ratio is enhanced by $40 \mathrm{~dB}$.

A subsequent selection from the data allows an analysis of the distribution of $B_{\text {eif }}$ at fixed time instants of the heart cycle. The baseline of the MCG is taken as zero level. This level is determined by averaging the values during a period of no electrical activity. In an arbitrary point on the map $B_{\text {eff }}$ is calculated by means of linear interpolation between the values in the grid points.

\section{5. - Results.}

Some results for healthy subjects are given in the accompanying figures. The maps are taken at various time instants throughout the heart cycle and 
are shown sequentially. The instant of time is indicated by the vertical line intersecting the inserted ECG.

In fig. 4 three types of isofunction plots recorded for 6 time instants are displayed for subject $A$. These plots are given in a separate column for each time instant. The boundary lines on the left and right of the isopotential body
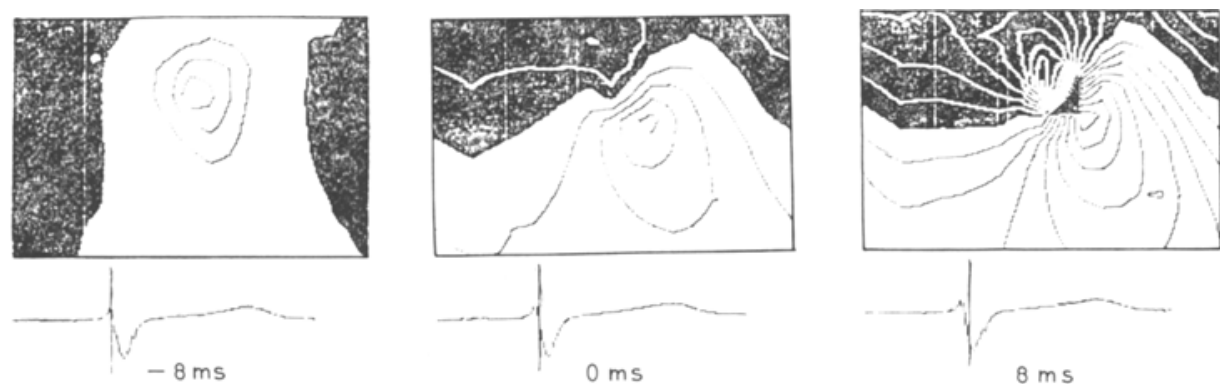

$8 \mathrm{~ms}$
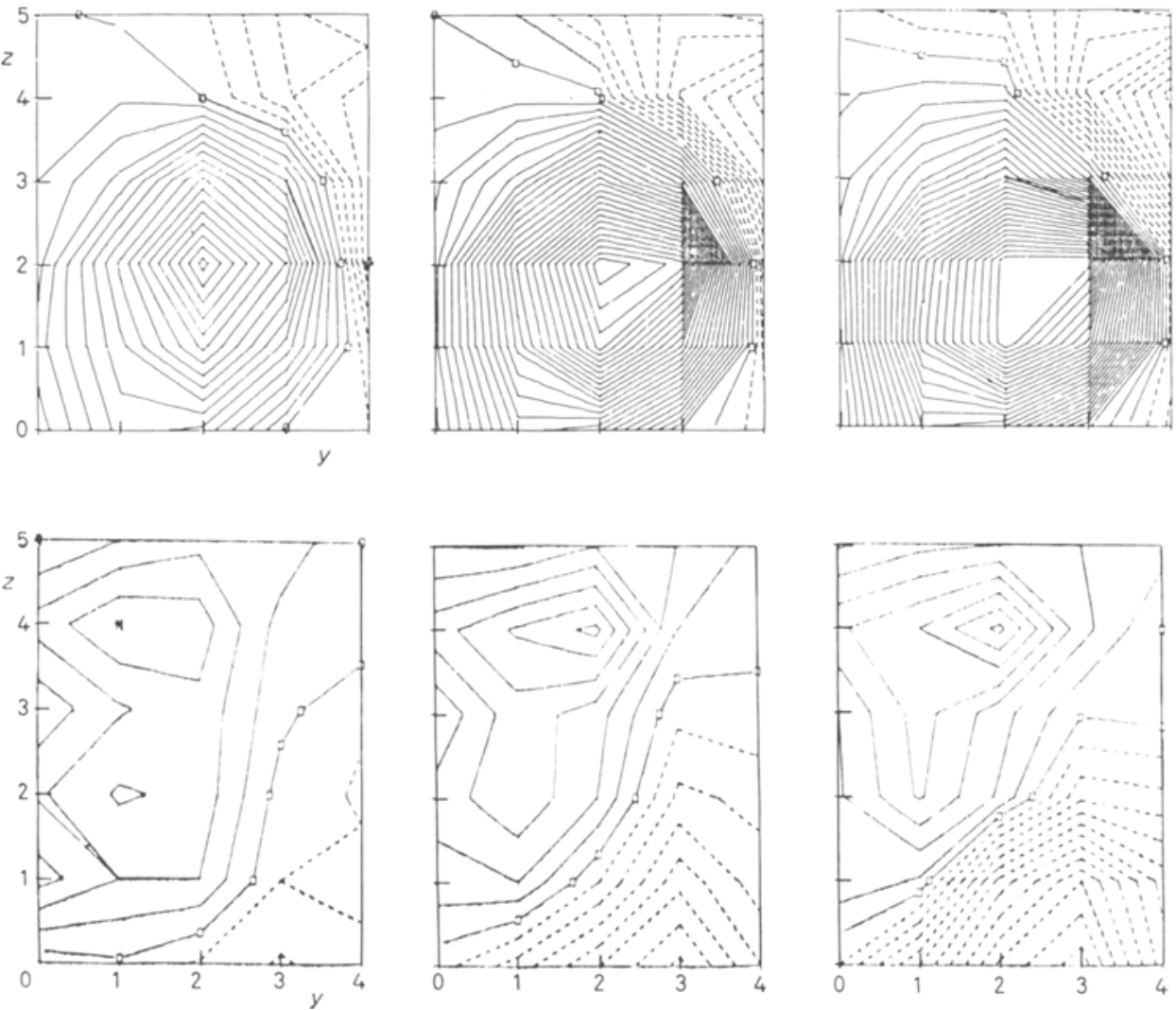

Fig. 4. - Isofunction plots of subject A. Upper row: isopotential maps; second row: isomagnetic field component maps taken from A's chest; third row: isomagnetic field component maps taken from A's back. 
surface maps, given in the upper row, are over the spine (see fig. 5). The dark regions represent negative values, the blank ones positive balues. The distance between successive lines is $0.2 \mathrm{mV}$. The isomagnetic field component maps which are displayed in the second row are based on measurements from A's chest. Solid lines indicate positive values, dashed lines negative and solid lines with small squares zero values. The distance between successive lines is

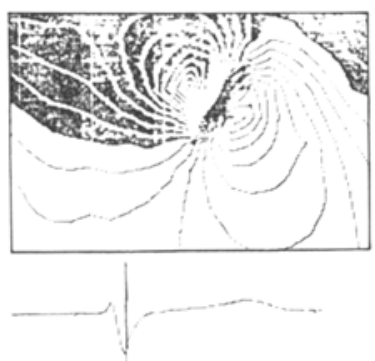

$24 \mathrm{~ms}$
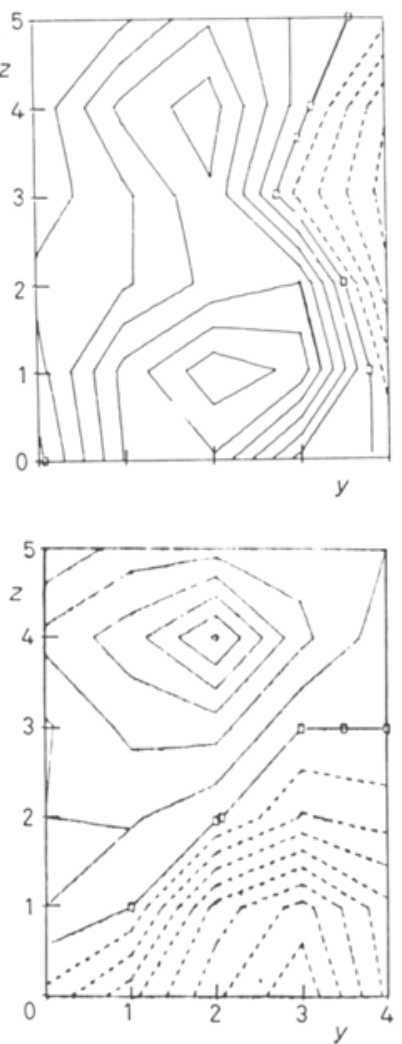

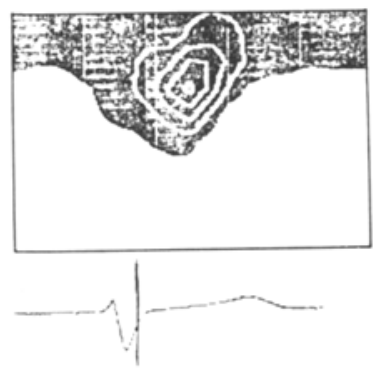

$40 \mathrm{~ms}$
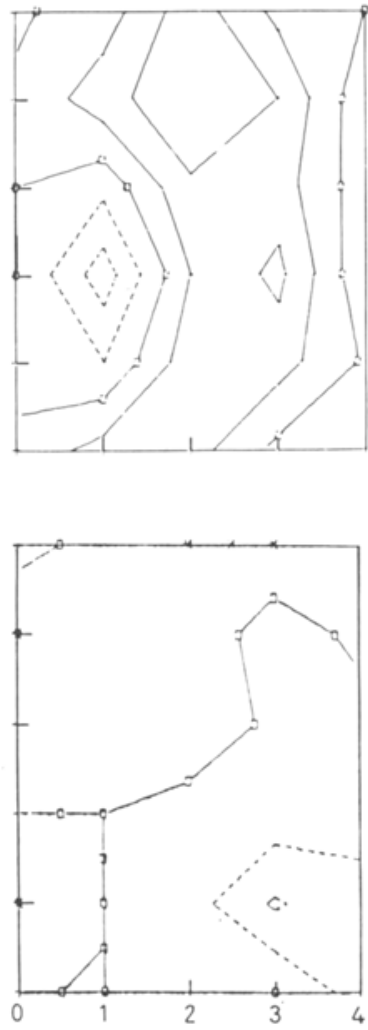

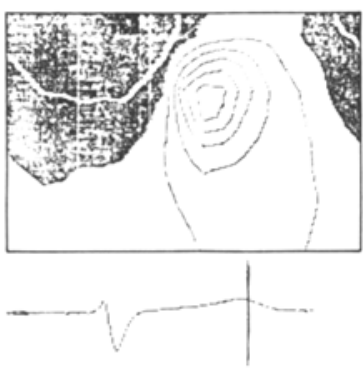

$260 \mathrm{~ms}$
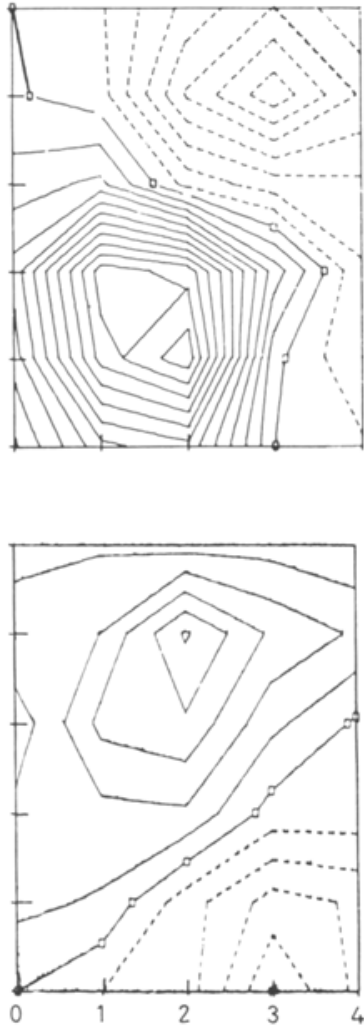

Fig. 4. - (continued). 


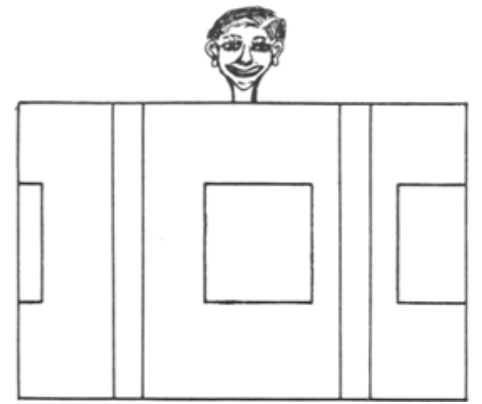

Fig. 5. - Projection plane of the body surface as employed in the isopotential maps. The location of the MCG map areas is indicated.

$7 \cdot 10^{-11} \mathrm{~T}$. The scale is $4 \mathrm{~cm}$ to one division. The point $(1,1)$ is over the lower end of the sternum. The isomagnetic field component maps which are displayed in the third row are based on measurements from A's back. The distance between successive lines is again $70 \mathrm{pT}$. A posterior view is given, where point $(3,1)$ is above the spine opposite to the lower end of the sternum. In fig. 6 conventionally represented MCGs taken from A's chest are shown. Figure 7 shows

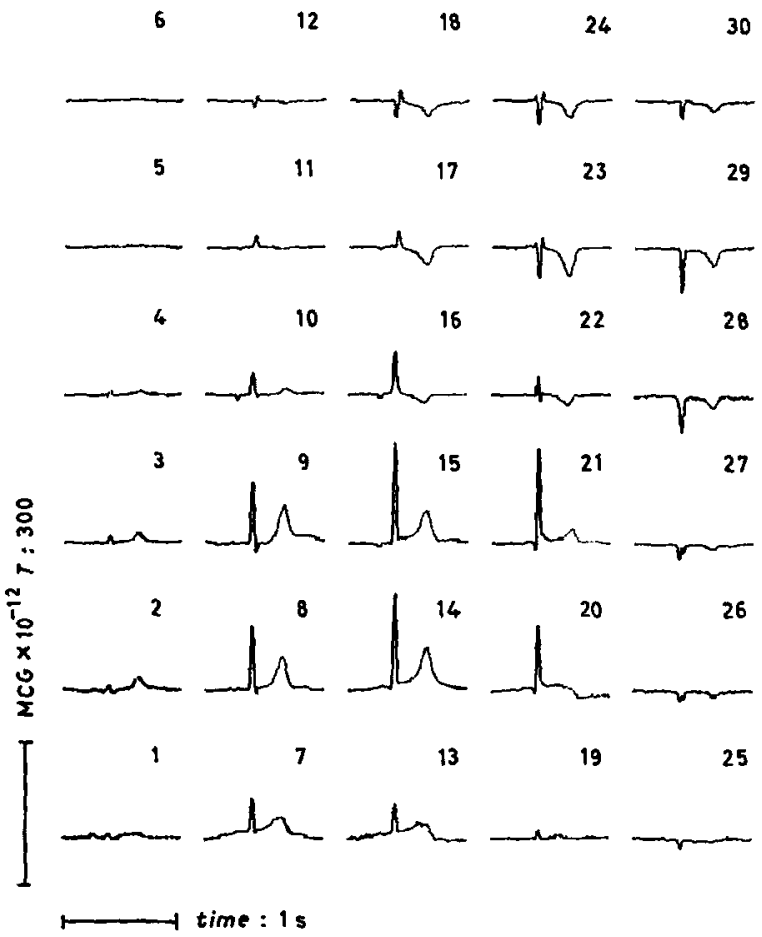

Fig. 6. - Magnetocardiograms as used in the construction of the maps in fig. 4 taken from A's chest. 

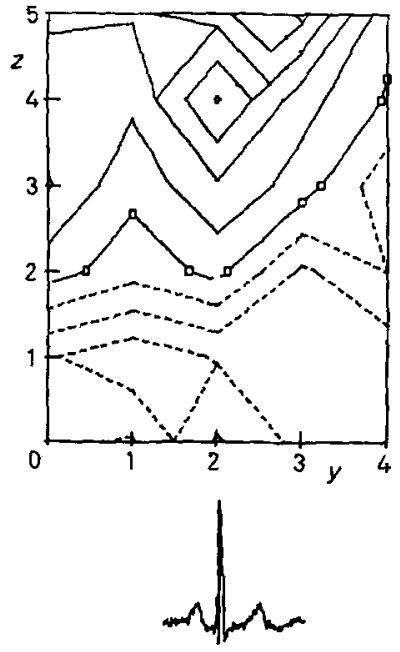

$-8 \mathrm{~ms}$

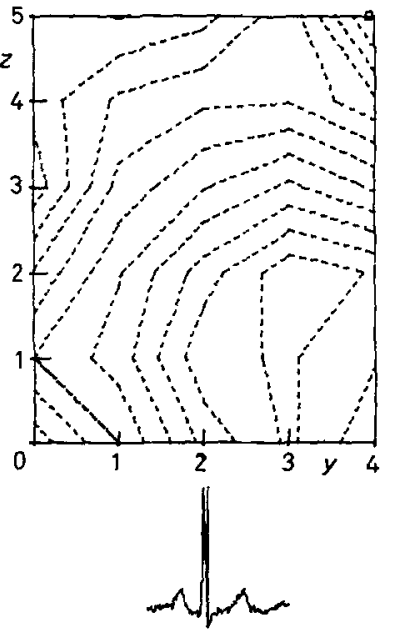

$24 \mathrm{~ms}$
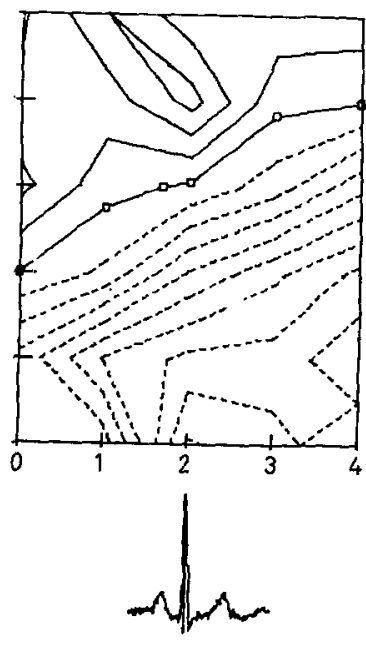

$0 \mathrm{~ms}$
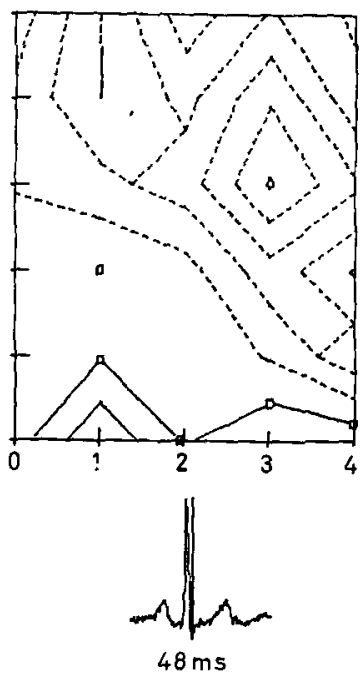
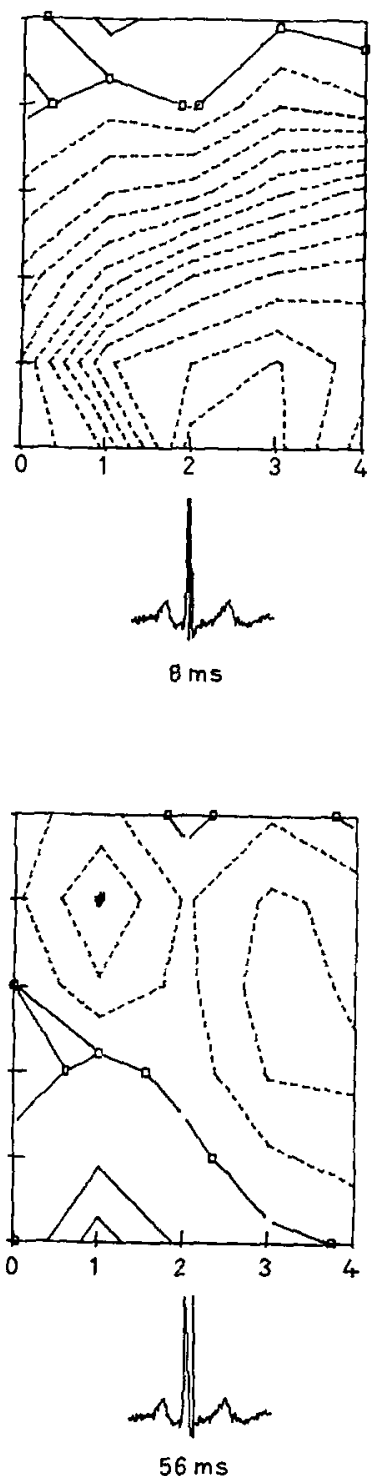

Fig. 7. - Isomagnetic field component maps taken from B's back.

isomagnetic field component maps from B's back. The distance between successive lines is $21 \mathrm{pT}$.

\section{6. - Discussion.}

The ratio between the amplitudes at the back and front is high for the magnetic field in comparison with the equivalent ratio for the electric potential. 
This high ratio is confirmed by numerical computations $\left(^{8}\right)$. For subject $B$ it turned out that the amplitudes shown in the maps which were taken at the back were almost equal to those taken at the chest during the time interval of $(0 \div 16)$ ms. The magnetic field contributed by a source decreases rapidly in strength with the distance from the point of observation. Near the ehest the maps are predominantly generated by the sources of the anterior part of the heart, the maps near the back predominantly by those of the posterior part. This may provide us with a method to distinguish pathological heart conditions in the anterior section of the heart from those in the posterior section.

The features of both electric and magnetic maps are simple. Looking at fig. 4 we see that the isopotential map at $t=-8 \mathrm{~ms}$ shows a small magnitude and it seems to describe the potential caused by a single dipole which is pointing from heart to chest. The magnitude increases during the time interval from $t==-8 \mathrm{~ms}$ to $t==8 \mathrm{~ms}$ and the dipole seems to turn into a frontal plane. During this time interval the magnitude hardly changes in both isomagnetic field component maps and also the direction of the lines indicating the zero value remains stable.

At $t=0 \mathrm{~ms}$ and $t=8 \mathrm{~ms}$ the lines indicating zero are in accordance with the simple theory mentioned previously, i.e. the zero line in the magnetic maps is perpendicular to that in the isopotential maps. The direction of the zero line in the map taken from the back is almost the same as the one taken from the chest. The behaviour shown (the features as one caused by a single dipole in a frontal plane whose direction is almost constant during the time interval from $t=-8 \mathrm{~ms}$ to $t=16 \mathrm{~ms}$ ) is representative for all our isomagnetic field component maps measured up till now (a total number of 10).

In the interval from $t=8 \mathrm{~ms}$ to $t=24 \mathrm{~ms}$ the isopotential maps and isomagnetic field component maps taken at the back are stable, though in the isofunction plot of $B_{\text {eff }}$ taken at the chest the features are changed a lot and the magnitude is decreased. This process started at $t=16 \mathrm{~ms}$ and this dramatic change occurs in all our maps taken at the chest.

The isopotential map at $40 \mathrm{~ms}$ shows the character of a map caused by a dipole pointing to the chest and indeed the contribution to $B_{\text {eff }}$ is very small in both isomagnetic field component maps. On the other hand, at $t=-8 \mathrm{~ms}$, at which the isopotential map shows the same characteristics (apart from the sign), the isomagnetic field component maps indicate the character of a map caused by a dipole in a frontal plane. Moreover, when the $T$-wave has its peak value at $t=260 \mathrm{~ms}$, the magnetic maps in fig. 4 show the character of one caused by a dipole in a frontal plane, while the isopotential map seems to be a reflection of a dipole with components in three orthogonal directions.

(8) M. J. Peters, M. J. M. Swennenhuis, A. van Oosterom and J. J. WeversHENKE: The influence of inhomogeneities on the cardiac-magnetic-field distribution in Abstracts Workshop on Biomagnetism Rome, 1982, to be published. 
We may conclude that the description of the current generators in the heart by a single current dipole is not a good approximation, although looking at only one type of map strongly suggests that such an approximation is quite reasonable at certain time instants. The finding that potential maps during the time interval discussed in this paper do not change in time, while the magnetic maps change considerably, indicates that the electric field and the magnetic field do reveal different information. A possible reason for this independence is the anisotropic nature of the cardiac tissue. For instance, the fact that the resistance along cardiac fibres differs from that across the fibres will have a larger effect on the magnetic field than on the electric field. This follows from model studies in which a dipole layer is modelled as an oblate spheroid with a nonuniform eurrent dipole density $\left(^{5}\right)$. MCG data could contain really new information due to vortex sources. WIKswo $\left(^{9}\right)$ suggested that the spiral geometry of the fibres may act as a vortex source. The usefulness of the reported independence for diagnostic purposes is not yet known. We intend to continue the mapping of both electric potential and magnetic fields, also in pathological cases.

The authors would like to thank Prof. Dr. L. C. VAN DER MAREL and Mrs. J. J. Wevers-Henke for their helpful discussions and Mssr. VeenstraLoKIN and J. A. ULFMAN for their technical assistance.

$\left.{ }^{(}\right)$J. P. WIKswo jr.: Proceedings of the Nato Advanced Study Institute on Biomagnetism, Frascati, 1982, to be published.

- RIASSUNTO(*)

Si registra la distribuzione sia del potenziale elettrico cardiaco sulla superficie del corpo che del campo magnetico cardiaco vicino al torace e alla schiena in momenti fissi del ciclo cardiaco. Le caratteristiche generali di entrambi i tipi di mappa sono semplici e riproducibili, inoltre ciascuno di essi fornisce informazioni differenti.

(*) Traduzione a cura della Redazione. 